\title{
TOWARDS SOCIALLY SUSTAINABLE WATERFRONT URBAN REGENERATION: THE CASE OF ZAYED PORT DESIGN, ABU DHABI
}

\author{
BARAAH MOUTAZ HAMDOON \& KHALED GALAL AHMED \\ United Arab Emirates University, United Arab Emirates
}

\begin{abstract}
While the urban waterfront is crucial to shaping the city image, it also contributes to the quality of life of the city's inhabitants and visitors. The Waterfront of Abu Dhabi city, the capital of the United Arab Emirates (UAE), has witnessed influential changes in the last 20 years. This research aims at discussing the relationship between urban form and social sustainability in a very recent urban regeneration of a waterfront project in Abu Dhabi city, the Zayed Port's waterfront project. The attainment of the social sustainability principles in the urban regeneration project was explored through examining the existence of these principles through an established theoretical framework. The theoretical framework included the main social sustainability principles of compactness and density, mixed-use and social mix, mobility, equitable access and spatial integration, safety and security, identity of place, and community participation. The utilized qualitative/quantitative tools of the case study method encompassed Space Syntax analysis of the urban form through relevant DepthmapX simulation variables. The assessment results revealed the gaps and potentials related to the application of these principles in the proposed design of the urban regeneration waterfront project of Zayed Port. Based on these outcomes, a set of social sustainability enhancement strategies has been recommended. The applied method with its tools as well as the revealed outcomes is envisaged to be significant to consider in the current and future urban regeneration designs of waterfront projects in the UAE's cities distinguished with their long waterfronts such as Dubai, Sharjah, Ajman, and other cities, to assure the achievement of social sustainability principles in this important type of urban development.
\end{abstract}

Keywords: urban regeneration, social sustainability, waterfront, Zayed Port, Abu Dhabi, UAE.

\section{INTRODUCTION}

Urban areas are always in a state of physical, social, environmental, and economic transformation [1]. Some of these transformations lead to a state of urban degradation that requires urban regeneration process to deal with. The practice of urban regeneration starts with identifying the major areas of concerns and the possible future challenges, then, it tackles the physical conditions enhancement, social improvement, and economic progress of the targeted urban area [2]. Waterfront urban development and regeneration, in specific, are representing valuable endeavor for urban communities stemming from common aspects of old ports areas such as the availability of vacant land and the potential of attracting new investment and cultural development. All over the world, various waterfronts urban areas have been regenerated from brown fields to commercial, residential, and recreational areas. While the main attention has been paid to the environmental and economic dimensions of most of the urban waterfront regeneration plans, the social dimensions have recently become increasingly important in urban policies [3].

Since its establishment as a federal state, the United Arab Emirates (UAE) has witnessed continuous urban development efforts which recently aimed at achieving sustainable development. Abu Dhabi, the capital city of the UAE, with its port and long waterfronts has been in the center of the contemporary efforts of waterfront regeneration in the country. Waterfronts in the UAE lay by the coast of the Arabian Gulf and have been 
historically considered the oldest sources for economic activities of the Emirati residents including trade, pearling, and fishing. With the aim to facilitate better living conditions, many of the current urban regeneration projects in Abu Dhabi city appear beside the water.

Abu Dhabi Urban Planning Council has issued the Abu Dhabi Public Realm Design Manual in which a chapter is dedicated to the urban planning 'guidelines' related to the Waterfront development [4]. As shown in Table 1, these guidelines define the needed amenities for waterfront development hierarchy with the overall aim to assure high-quality waterfront developments, make them accessible destinations for both residents and visitors, and provide diverse public space designs that celebrate and strengthen local heritage of Abu Dhabi. Table 1 illustrates the guidance for the types of the served users, the needed features and/or amenities, the service radii, and the level of service that provides benchmarks for the percentage of the accessible waterfront frontage for each hierarchy level [4].

In this research, a conceptual design alternative for Zayed Port in Abu Dhabi city was assessed from a social sustainability point of view to define the shortcomings and potentials associated with the design and to provide future insights for enhancing the attainment of socially sustainable urban waterfront regeneration projects, not only in this and other similar projects in the city, but also in other waterfront cities in the UAE.

\section{RESEARCH PROBLEM AND METHOD}

While the waterfront urban regeneration is receiving global attention from researchers and scholars, locally in the UAE, there is almost no literature that have reviewed the sustainability of recent local waterfront communities in the country, especially from a social sustainability point of view. Usually, social impacts have contextual features, and they represent complex social relations or dynamics. The Zayed Port Pier urban regeneration design [5] was selected as the case study of this research as it is one of four major ports in the Emirate of Abu Dhabi and it is a prominent project reflecting the very recent trend in considering sustainability in waterfront urban regeneration in the country.

Established in 1968, Zayed Port is located in the northeast section of Abu Dhabi city (Fig. 1). The Port covers an area of about 535 hectares, with 21 berths and a total berth length of 4,375 meters. The port is characterized by its containment of a traditional Dhow harbor, and its continuous fish trading tradition of earlier times. The site is overlooking the Al Lulu Island, and the port is located beside Al Corniche from the South and the Southwest directions. While from the Northwest direction the port is overlooking the Louvre Museum in Al Saadiyat Island.

The design of the urban regeneration of the Zayed Port Waterfront Pier by the Sasaki Architects [5] is conceived as a fully integrated mixed-use district tied by a series of pedestrian-oriented public spaces and a world-class waterfront promenade. As shown in the mixed land-use plan of the design (Fig. 2), a circular-shape hotel complex anchors the west end of the redevelopment site and overlooks Abu Dhabi city, Al Lulu Island, and the Arabian Gulf, while the middle blocks of the redevelopment plan combine three hotels, meeting and ballroom facilities, retail spaces, and residential apartments into one district. Besides its local residents, expectedly from middle class stratum, the Zayed Port Waterfront Pier project is intended to serve a mixture of users including tourists, visitors from surrounding areas. Also, the plan will offer various jobs opportunities for all factions of the society.

To assess the Zayed Port Pier urban regeneration design from a social sustainability perspective, the research first initiated a theoretical framework for the main principles and indicators of socially sustainable urban waterfront regeneration. This theoretical framework 


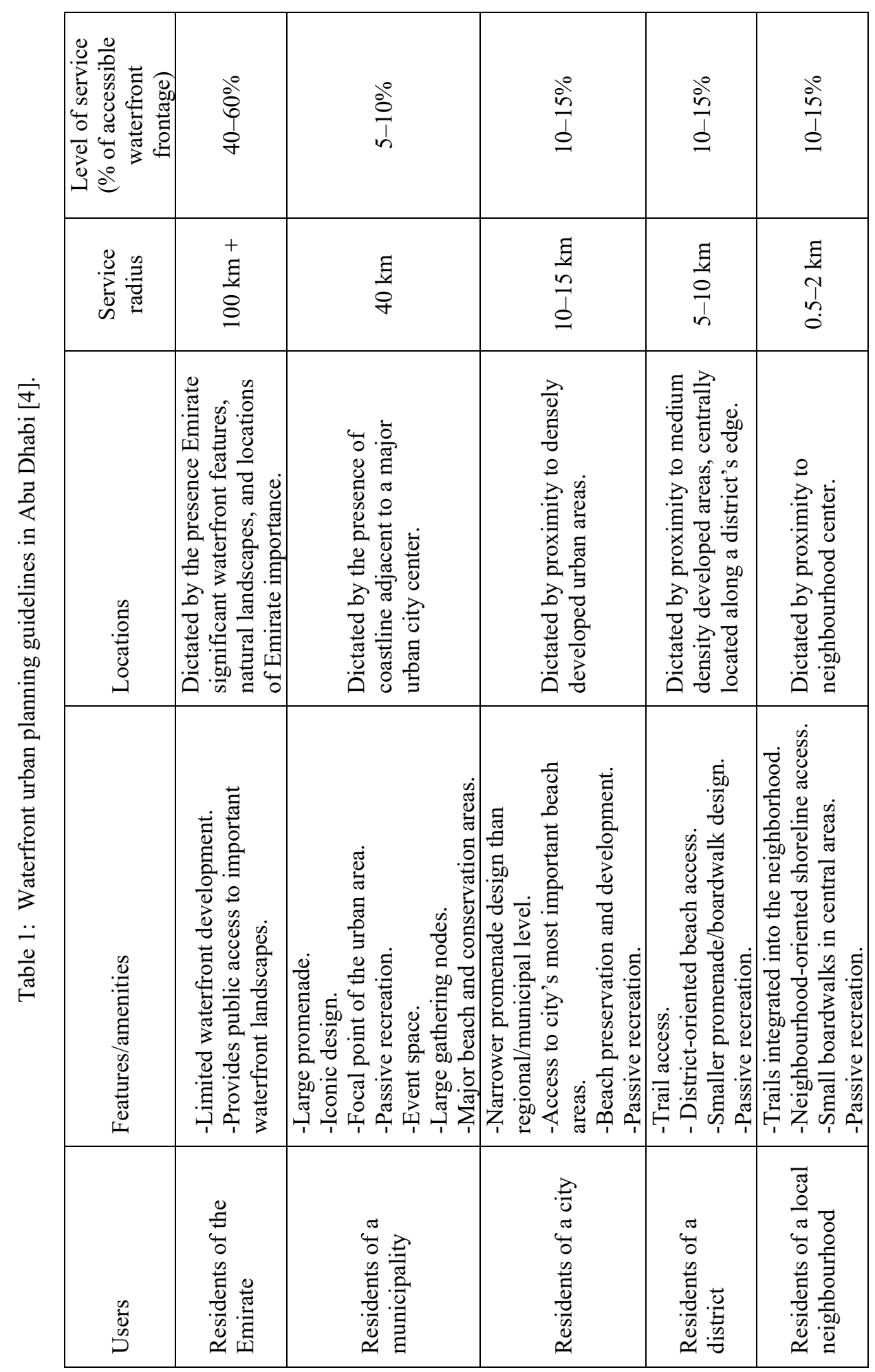



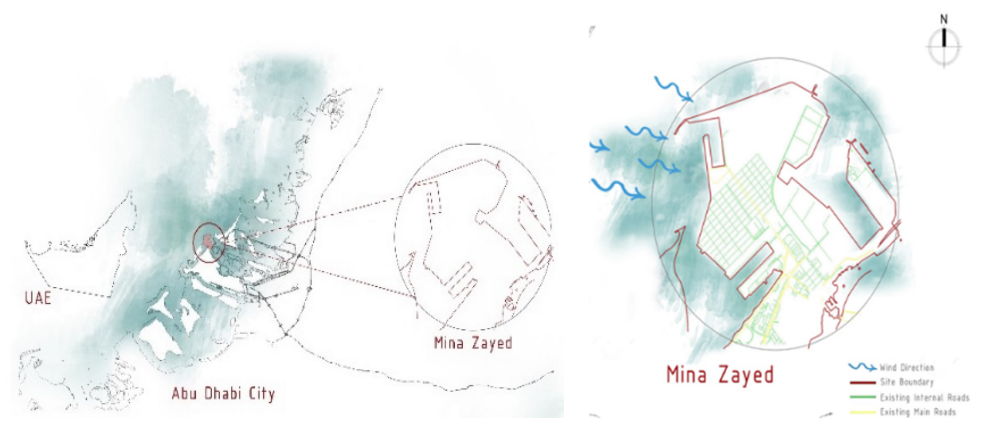

Figure 1: The location of Mina Zayed in Abu Dhabi City.

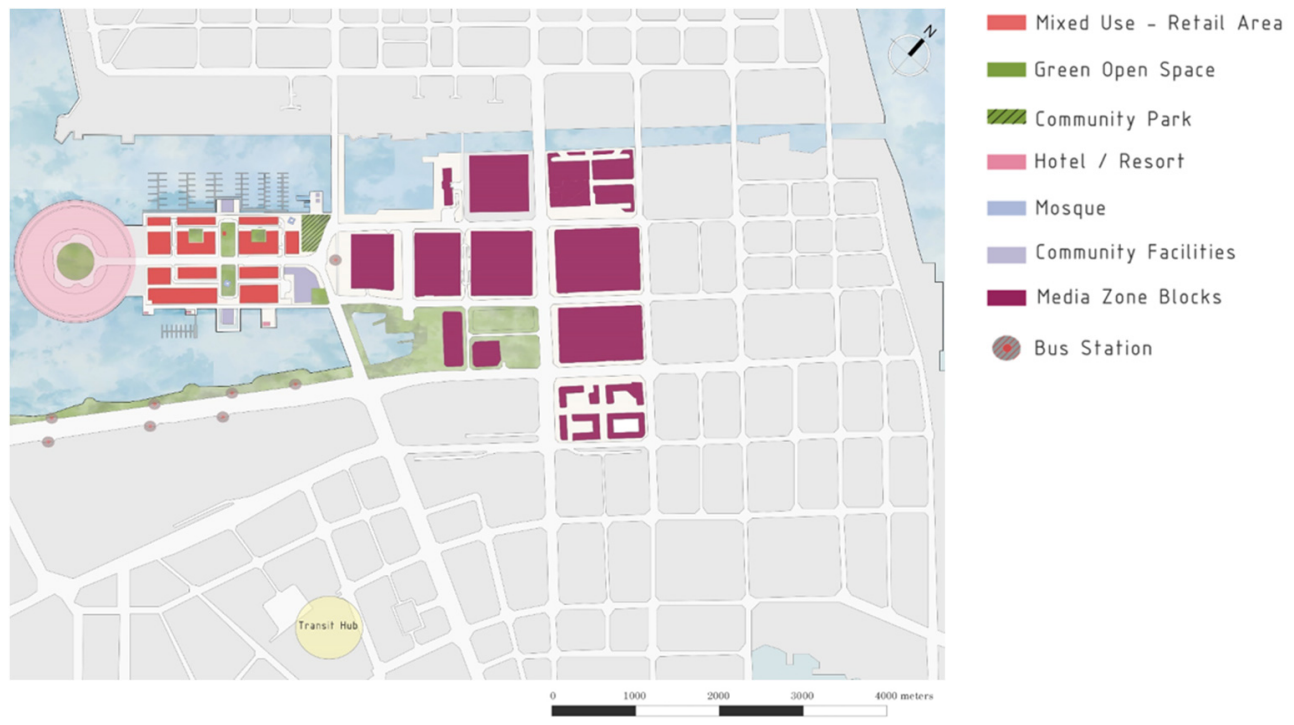

Figure 2: The Land-use Plan of Mina Zayed Waterfront Pier - the development of the services designed by Sasaki Architects. (Source: Based on [5].)

was mainly derived from two sources: the relevant global literature, and some relevant case studies. Then, this framework was used to assess the proposed design of Zayed port and ultimately a set of future design guidelines and recommendations has been developed out of the results of this assessment. In terms of the main investigation tools of the applied case study method, the design CAD drawings of the Zayed Port urban regeneration project were explored and analyzed against the defined target social sustainability principles using quantitative/qualitative tools especially Space Syntax analysis through DepthmapX simulation software, as a reliable tool for investigating social impacts of urban forms [6]. In DepthmapX software, the produced Integration values for the whole urban system (Rn), calculated through Angular Segment Analysis, were used to assess the spatial integration measures of the design. Meanwhile, Choice values were used to assess accessibility measures of the urban form of the design. Meanwhile, catchment distances were calculated using the values of Step Depth which follow the shortest angular path from the selected 
segment to all other segments within the whole urban system [6]. In addition, some qualitative tools were applied, as relevant, to probe some qualitative social sustainability principles and indicators of the waterfront urban regeneration such as Identity and Sense of Safety and Security.

\section{PRINCIPLES OF SOCIALLY SUSTAINBLE WATERFRONT URBAN REGENERATION}

In general, the social dimensions of urban waterfront planning and regeneration refer to the different ways of experiencing and using the edges of sea to make an understanding of their qualities for the community. To initiate a matrix of principles for socially sustainable waterfront urban regeneration that could be used to assess related designs, the research consulted a wide variety of relevant literature, local guidelines, and some case studies aiming at developing a comprehensive set of relevant principles and indicators. Still, due to the nature of the literature about social sustainability, this conceptual framework might have unintentionally missed out some of the principles and/or indicators. As briefly detailed below, these principles included main seven principles as follows: Compactness and Density, Mixed-Use and Social Mix, Mobility, Accessibility, Safety and Security, Identity, and Community Participation.

First, Compactness and Density of the built environment is a widely acceptable strategy through which more sustainable urban forms could be achieved. This is because compactness leads to urban connectivity, which minimize the need for transport of people, products, and materials. It also leads to less use of urban land due to increasing the density of development and activity [7]. The Floor-Area-Ratio (FAR), calculated by dividing the total floor areas of the buildings by the gross area of the development site, is suggested as a measure of urban form compactness and indicating the development density [8].

Second, Mixed-Use and Social Mix enhance the condition of social sustainability through giving the residents the opportunity to meet their needs within their locality. In this way, the people would become less dependent on private automobile trips and will be more encouraged to walk toward their desired destinations. This would also strengthen the social ties and networks among the residents through stimulating their interaction in a way that enhances the life quality and social equity of the local community [9]. On the other hand, social mix and mixed land-use are interdependent and promote each other. Actually, social mix aims to encourage the interaction between different social stratum in the same community and to ensure the accessibility to equitable urban opportunities. Social mix helps to provide the basis for healthy social networks and social capital, which are the driving force of socially sustainable communities [10].

Third, Mobility, with the provision of its different modes including walking, cycling, public transport, and private vehicles, is an essential social sustainability principle. It is important for a socially sustainable built environment to encourage walking and cycling through providing a safe, attractive, and well-maintained walkways and cycling networks. The interconnected public realm enhances the mobility of people by providing comfortable and continuous access for walking, cycling, and other modes of transport with their different design strategies [4]. One of the most important services which highly contributes to the neighborhood's level of mobility is public transportation. While there are different types of public transportation - i.e., train, tram, bus -, their applicability depends on various factors including the population and/or development density [11]. Meanwhile, private cars mobility network should be allowed but with less permeable configuration and with applying traffic calming measures to control the impact on pedestrian and cyclists [12]. 
Fourth, Accessibility is highly required in waterfront developments for their attractive and vibrant public destinations. Within the developed waterfront area itself, catchment distances to locally provided services and public transportation nodes between $400 \mathrm{~m}$ and $800 \mathrm{~m}$ are recommended. Furthermore, it is better to centrally locate the local services and facilities and to be near to a main transport node. Ease of access into local services and facilities for elderly, children and disable people, should also be considered. The stop intervals of public transport nodes within the development area should be within a $t$ distance of about $200 \mathrm{~m}-300 \mathrm{~m}$. On the other hand, the waterfront development should have Spatial Connectivity and Integration both internally and externally with the surrounding urban context. Integration could be achieved through a reasonably permeable grid that results in linkage of the urban spaces [13].

Fifth, Safety and Security entail the provision of safe, secure, and accessible spaces for all members of the waterfront development residents and visitors. Compact and inclusive urban development plans could cater to security and safety measures for all. Additionally, traffic calming measures, visual surveillance, and the provision of a safe and connected mobility networks should all be achieved in public areas through the urban development plan [14].

Sixth, Identity represents what is unique about a community's culture, heritage, and natura assets, in the development urban and architectural design of buildings, streets, streetscapes, green spaces, plantings, etc. Outstanding features of natural and man-made heritage of the developed waterfront area should be considered in the design [4].

Seventh, Community Participation is acknowledged in the contemporary planning development as it helps accomplish a sustainable development design. As the professionals and users are two quite different groups in terms of educational background, ways of thinking, "taste," and many other aspects, a participatory process can bridge this gap resulting a design based on an adequate understanding of the public's needs and preferences [15], [16].

\section{SOCIAL SUSTAINABILITY ASSESSMNET OF THE DESIGN}

Density and Compactness: The overall site area is about 51 hectares and the total floor area of the buildings in the urban regeneration design is about $560,000 \mathrm{~m}^{2}$. The calculated floorarea-ratio (FAR) of the urban regeneration design, as a measurement of the urban form compactness of the project, is 1.65. This figure indicates a high dense and compact value.

Mixed-Use and Social Mix: The masterplan of the Zayed Port Waterfront Pier project is a compact mixed-use development, which consists of residential, commercial, and service facilities. The mixture of uses includes three hotels, meeting and ballroom facilities, retail space, apartment blocks, green open spaces, community facilities, and media zones, as illustrated in Fig. 3.

Still, some of the required services and facilities, such as schools, clinics, and toddler play areas, are not provided in the master plan of the project. On the other hand, social mix is expected due to the diversified services and facilities in the project as discussed earlier. Residential dwellings are also provided with a variety of areas but are apparently targeting the requirements of middle-class social stratum.

Mobility: The Zayed Port Waterfront Pier masterplan connects the different provided facilities in the site from Northwest, Northeast, and South directions by convenient pedestrian walkways and footpaths that integrate the whole waterfront redevelopment area (Fig. 4). Additionally, the design of the 5-meter-wide pedestrian lanes in the main streets are mostly shaded and artificially lit following a typical city Avenue urban design typology. 


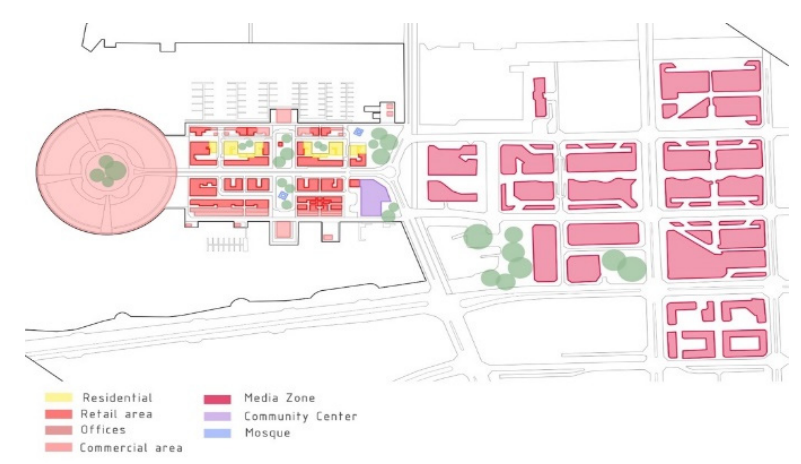

Figure 3: The ground floor plan of Zayed Port Waterfront Pier. (Source: Based on [5].)

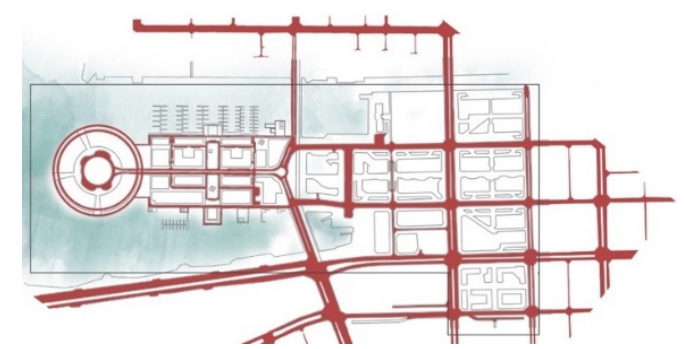

Figure 4: The Zayed Port Waterfront Pier Street pedestrian access [5].

Dedicated cycling lanes are not considered in the masterplan, but they are shared with pedestrian lanes. The design is not showing dedicated facilities and parking areas for cycles. For Public transportation, the proposed tram will link Zayed Port facilities to the designed multi-modal transit facility in Abu Dhabi city. Visitors who will arrive by tram to the Transit Plaza of the Waterfront Pier can easily then be guided to points north and south along the pier promenade. There is only one main line for public transportation which is serving the whole Waterfront Pier project. Meanwhile, bus stops are not available indie the project site.

Accessibility: The services and facilities in the Zayed Port Waterfront Pier project are open for the public with no restrictions. For the walkable catchment distances, the Step Depth Angular Segment Analysis in DepthmapX simulation tool show variant values for the different provided services and facilities in the study area. Fig. 5 illustrates two of these simulations results for the community parks and mosques.

About $60 \%$ of the site is not within the $400 \mathrm{~m}$ catchment distance for the community parks located in the Northwest of the project. Almost the same result is recorded for the mosques. On the other hand, the $800 \mathrm{~m}$ catchment distances for the local shops, retails, restaurants, and public services seems mostly achieved. For the accessibility analysis of the public transportation nodes (Fig. 6), the locations of the bus stops are not easily accessible by most residence of the project, as they are located along the corniche (Fig. 6(a)). Fig. 6(b) illustrates the catchment distance simulations, using Step Depth Analysis in DepthmapX, for the Transit Hub, tram stops, and bus stops, in and around the Zayed Port Waterfront 


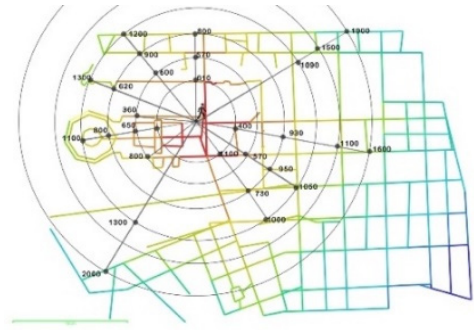

(a)

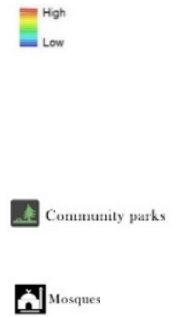

国

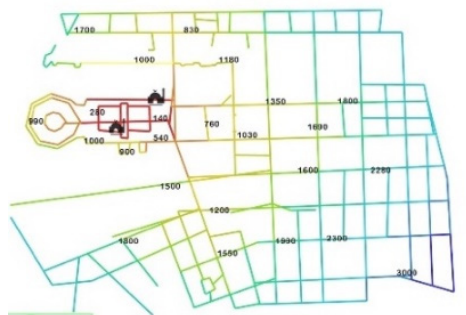

(b)

Figure 5: Segment analysis-step depth: The accessible distance for the community park and Mosques in The Zayed Port.

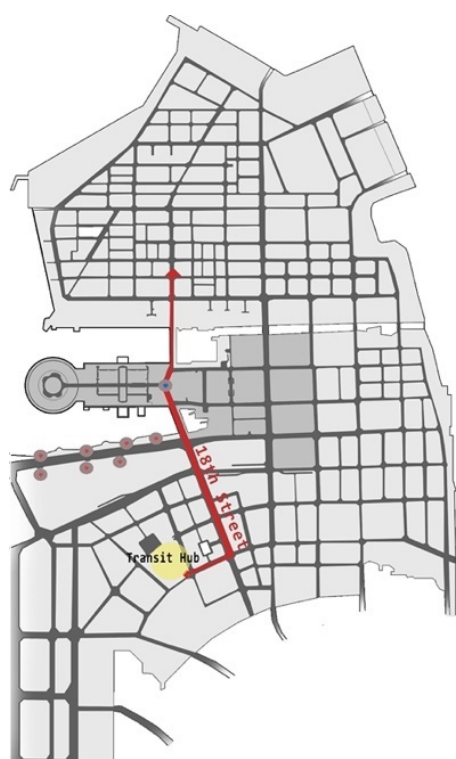

(a)
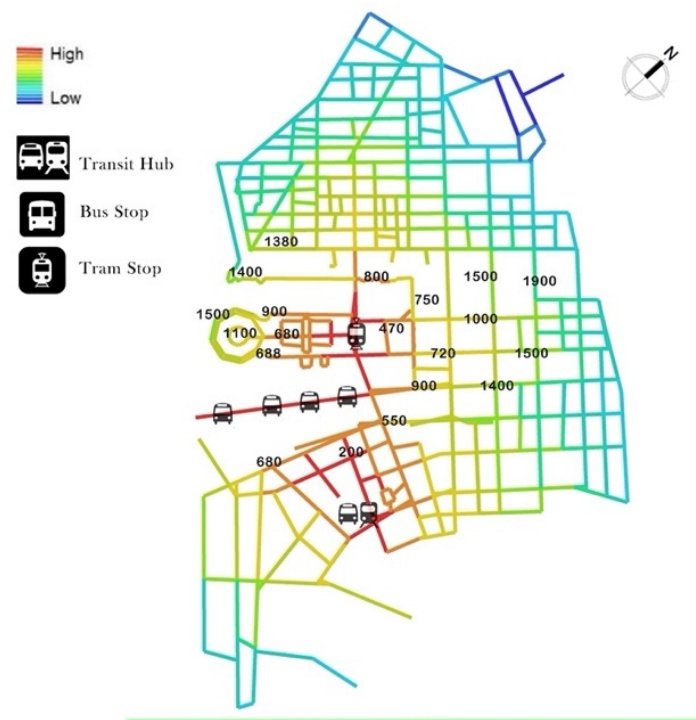

(b)

Figure 6: (a) Public transportation access from Transit Hub; and (b) Segment analysisstep depth: The accessible distance for the transit nodes.

Pier and other surrounding segments within the urban system. Unfortunately, it is revealed that about $20 \%$ of the urban regeneration area is not located withing the walkable catchment distance of 600 to $800 \mathrm{~m}$.

Accessibility requires spatial connectivity and integration among the residential, commercial, recreational, and civic uses of the project. The integration values were analyzed in DepthmapX for the whole urban system (Rn) (Fig. 7(a)). The obtained values show that the roads in the Waterfront Pier project are not perfectly integrated, where the main arteries around the basic services and facilities have recorded low integration values, while the most integrated road is located far from the mixed-use area. This makes the public services and facilities in the Southwest part of the project are not easily accessible by the visitors. 
Additionally, the availability of accessibility routs leading to the designed services and facilities within the study area was analyzed through simulating the Choice values in DepthmapX software for the whole urban system (Rn). According to Fig. 7(b), the most "chosen" arteries (highlighted in red) are the one located between the media zone and the community center, and another one located in the Northeast side of the Waterfront Pier. Both roads are far from the mixed-use areas of the project.

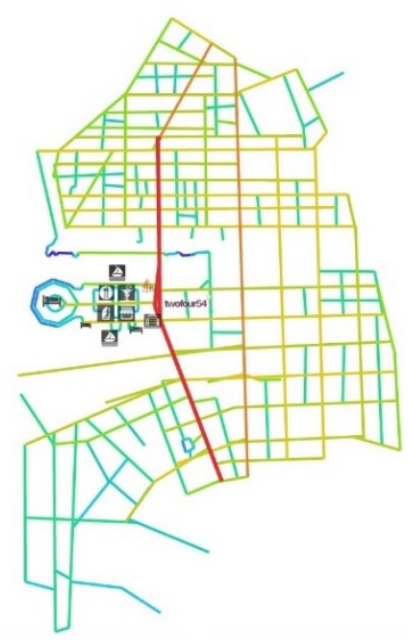

(a)

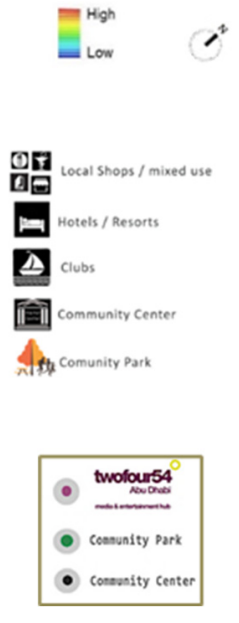

Figure 7: Angular segment analysis: (a) Integration-Rn, and (b) Choice-Rn.

Safety and Security: The design of the streets considers the provision of safety buffers to isolate pedestrian walkways from vehicular movement but, on the other hand, there are no separated cycling paths, which is a safety concern. Visual surveillance in the public realm would be positively affected by the provision of mixed-use mixed commercial and residential areas where the shops ensure a sufficient population in the street in the daytime and the residential use ensures a natural surveillance during the nighttime. The masterplan is designed to in a way where public spaces are easy to overlook and to oversee. But with the lack of appropriate catchment areas, residents' presence in the streets might not be enough for the provision of visual surveillance. On the other hand, the measures of inclusive design that consider the needs of children, the disabled, and elderly people are mostly achieved in the design. The good consideration of visibility through effective artificial lighting enhances the sense of security.

Identity: Whereas the Zayed Port location contains important natural and heritage assets of the Arabian Gulf, the design seems not considering these assets sufficiently to help create a distinctive identity either in the masterplan of the port design or the architectural design (Fig. 8).

Community Participation: Actually, the current proposal does not directly involve the community in shaping its vision and concepts. But, as mentioned by the designer, Sasaki's architect, the Zayed Port design helps the community to engage in various shared social activities through the provision of public spaces, plazas, tourism area, yacht club, public landmarks, and mosques. 


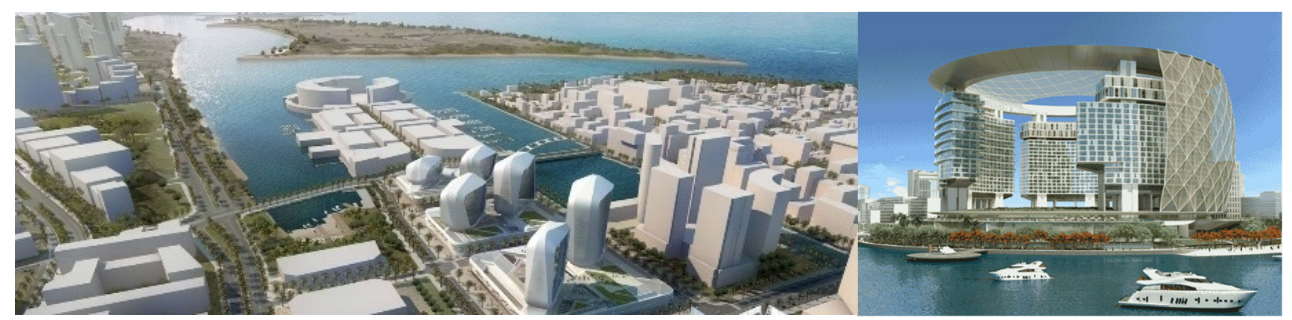

(a)

(b)

Figure 8: Zayed Port Waterfront Pier aerial view [5].

\section{DISCUSSION: RETHINKING THE DESIGN OF ZAYED PORT WATERFRONT}

The outcomes of the investigations managed to assess the defined social sustainability principles in the examined case study. This gives confidence in the applied method and tools which might help enhance the assessed design and any other urban regeneration design for similar waterfront development cases. For the case of the Zayed Port urban regeneration project, the assessment reveled 5 potential enhancement strategies that would make the regeneration design more socially sustainable. These are: first, redefining the land-use of the project to provide all the missing local community facilities and services in the design, such as clinics, schools, play areas, etc. In addition, the number of some provided facilities such as mosques, etc., need to be increased in some locations as revealed in the analysis, to cover the need of these services in all areas of the project. More variety of housing types is also needed. Traditional activities related to the Port, such as a fish market, could be added near to the Marina. As the project also attracts tourists and visitors, some city scale services such as a vegetable/fruit market, and handmade carpets market, might be good additions to the land-use of the design.

Second, improving modes of mobility by enhancing the pedestrian network, adding dedicated cycling infrastructure, and more importantly applying the relevant code for the streets' typical cross sections according to each street category (boulevard, Avenue, Street, Alley (Sikka), etc.) as per The Abu Dhabi Urban Street Design Manual [17]. Minimizing the cars' right of way would enhance other more sustainable mobility systems of pedestrians, cyclists, and public transportation. This would give the residents and visitors the ability to choose the mode of movement and commuting that best fits each one of them. In new urban districts, like the project in hand, it is preferable to place car parking and service delivery belowground to save the public street realm for use of people. Meanwhile, the streetscape design of the Zayed Port Waterfront Pier development should be designed to enhance thermal comfort by creating a network of safe, comfortable, and continuously shaded routes.

Third, improving accessibility through developing the design of the street network to be more integrated with the surrounding urban context. In addition, applying Transit Oriented Development through providing the services and facilities around the public transportation hub, would enhance accessibility. Enhancing pedestrian accessibility through meeting the required catchment distances might require adding dedicated pedestrian pathways, known as sikkas in traditional urban development of Abu Dhabi. To enhance accessibility to public transportation nodes, bus routes need to penetrate the redevelopment area with nodes within walkable distances to the residents. This will encourage a greater number of residents to consider bus/tram-trips as a viable solution to their travel needs. 
Fourth, enhancing visual identity of the project in a way that sustain the local culture of the Port by better reflecting the history of the waterfront area as an old Dhow harbor. The notion of 'authenticity' should be reflected in the urban form and building design that naturally blend daily activities with culture and the street life. Achieving this in the project requires more reliance on local urban forms, construction materials, open spaces that resonate with the local climate conditions and other distinctive attributes that are tied to local traditions of Abu Dhabi as a Port city. Moreover, adding dedicated spaces such as floating parks containing an array of seasonal activities, including areas for traditional performance, play, and quiet rest places, that are overlooking the Arabian Gulf, might also help enhance the visual identity of the project.

Fifth, enhancing the level of community participation by involving community members in the design decision-making processes of the project. Engaging the local public, as part of the design strategy, would allow people to be involved directly in shaping the project's visions and ideas, and consequently better design decisions will be reached.

\section{CONCLUSION}

The Zayed Port Waterfront urban redevelopment is perceived as a sustainable initiative, that will revive the old port district to become a new social and civic hub in Abu Dhabi city, i.e., a place where it is enjoyable for people to live, work, shop, visit, and spend time with family and friends. As social sustainability is a vital pillar in these types of projects, adhering to social sustainability principles is essential in the design to help restore the vibrancy and enhance the quality of life for people. By that the urban redevelopment project will be a place that is scaled to people, providing safe pedestrian environments and open space for vivid and safe public life. Most importantly, it will be a place grounded in the unique culture and values of its people and spatially integrated with the waterfront and the Port.

The study aimed at assessing the current urban regeneration design concept of the Zayed Port from a social sustainability point of view. Accordingly, a conceptual framework for the main seven principles of social sustainability and their indicators was briefly established and consequently used as a tool of assessing the original urban form of the urban regeneration design. The utilized qualitative and quantitative tools, especially Space Syntax simulation, proved valid in defining the shortcomings of the proposed design in relation to the examined social sustainability principles. These were related mainly to the mixed-use plan, modes of mobility, accessibility, safety and security, visual identity, and public participation. Based on the assessment findings, a recommended set of social sustainability improvement strategies has been identified encompassing valid, reliable, and highly contextual design guidelines that could meet the needs of people in this geographic and cultural context, and hence enhance their quality of social life and well-being. If applied, it is envisaged that these strategies would enhance the design of the urban regeneration project of the Zayed Port Waterfront to be a more socially sustainable project. In addition, appropriate consideration of social sustainability principles would mutually reflect on other sustainability measures. For example, the socially sustainable mixed-use design can improve the energy efficiency through the development of the public transportation node around the high-density areas and providing proper catchment distances for walking and cycling, etc.

Finally, it is hoped that the outcomes of this research would pave the way for futuristic urban design scenarios that will lead to a more socially sustainable urban forms of waterfronts developments in the UAE and maybe in other Gulf Cooperation Council 
(GCC), Arab, and Middle Eastern cities that share many socio-cultural and environmental circumstances and contexts.

\section{ACKNOWLEDGEMENT}

The authors would like to thank the Research Office at the United Arab Emirates for funding this research project UPAR - Grant No. G00003450.

\section{REFERENCES}

[1] Roberts, P., Sykes, H. \& Granger, R., Urban Regeneration, 2nd ed., The British Library: London, 2017.

[2] Roberts, P. \& Sykes, H., Urban Regeneration: A Handbook, SAGE Publications: California, 1999.

[3] Girard, F., Kourtit, K. \& Nijkamp, P., Waterfront areas as hotspots of sustainable and creative development of cities. Sustainability, 6(7), pp. 4580-4586, 2014.

[4] Abu Dhabi Urban Planning Council, Abu Dhabi Public Realm Design Manual, Abu Dhabi Urban Planning Council: Abu Dhabi, 2017.

[5] Sasaki Architects, Zayed Port Waterfront Pier. https://www.sasaki.com/project/146/ mina-zayed-waterfront-pier/. Accessed on: 12 Mar. 2020.

[6] UCL Space Syntax. https://www.spacesyntax.online. Accessed on: 2 Feb. 2021.

[7] Eizenberg, E. \& Jabareen, Y., Social sustainability: A new conceptual framework. Sustainability, 9(1), pp. 1-16, 2017.

[8] Cheng, V., Understanding density and high density. Designing High-Density Cities for Social and Environmental Sustainability, E. Ng (ed.), Library of Congress Cataloging-in-Publication Data: London, pp. 3-17, 2010.

[9] Jones, A., Urban waterfronts: Learning from legacies lessons from the past pointers for the future. Proceedings of the Conference Regenerating Maltese and Sicilian Waterfronts: Perspectives from Two Mediterranean Islands, Kalkara, pp. 1-16, 2013.

[10] UN Habitat, A New Strategy of Sustainable Neighborhood Planning: Five Principles, Discussion Note 3 Urban Planning. https://unhabitat.org/Wpcontent/uploads/5May2014-Principles_web.pdf. Accessed on: 22 Mar. 2020.

[11] Jabareen, Y.R., Sustainable urban forms their typologies, models, and concepts. Planning Education and Research, 13, pp. 61-173, 2006.

[12] Dempsey, N., Bramley, G., Power, S. \& Brown, C., The social dimension of sustainable development: Defining urban social sustainability. Sustainable Development, 19, pp. 289-300, 2011.

[13] Barton, H., Sustainable Communities the Potential for Eco-Neighbourhoods, Earthscan Publications Ltd: London, 2000.

[14] Lee, J., Sher, W., Lee, S., Mak, M. \& Ostwald M., Strategic planning indicators for urban-a case study on mixed-use development in Seoul. Proceedings of the 19th Annual Pacific-Rim Real Estate Society Conference, Melbourne, Australia, pp. 1316, 2013.

[15] Empel, C., The effectiveness of community participation in planning and urban development. Proceedings of the Sustainable City V, pp. 549-556, 2008.

[16] European Commission DG ENV, Social Dimensions of Urban Waterfront Planning, European Commission DG ENV News Alert, Issue 15, 2006.

[17] Abu Dhabi Urban Planning Council, Abu Dhabi Urban Street Design Manual, Abu Dhabi Urban Planning Council: Abu Dhabi, 2017. 